\title{
AVALIAÇÃO DO PERFIL DE DISSOLUÇÃO DA GLIBENCLAMIDA CRISTALIZADA POR PROCESSO DE PRECIPITAÇÃO COM ANTISSOLVENTE LÍQUIDO
}

\author{
M. C. ABRANTES ${ }^{1}$, C. S. D. $\operatorname{COSTA}^{1}$ e G. L. V. COELHO ${ }^{1}$ \\ ${ }^{1}$ Universidade Federal Rural do Rio de Janeiro, Departamento de Engenharia Química \\ E-mail para contato: coelho@ufrrj.br
}

\begin{abstract}
RESUMO - Para controle do diabetes mellitus do tipo não-insulino-dependente tem-se utilizado hipoglicemiantes orais contendo o princípio ativo denominado glibenclamida (GLIB). Tal substância, mesmo possuindo alta permeabilidade, apresenta baixa solubilidade em água o que faz com que sua taxa de dissolução seja a etapa limitante da absorção da droga no organismo. Por isso, fez-se testes de dissolução com a GLIB cristalizada através do processo de precipitação com antissolvente líquido, de modo a determinar a quantidade de droga presente no meio de acordo com o tempo em que é exposta ao mesmo, e consequentemente, avaliar seu perfil dissolução.
\end{abstract}

\section{INTRODUÇÃO}

O diabetes mellitus é uma doença que tem afligido uma parcela significativa da população mundial nos últimos tempos e que, de acordo com a OMS (Organização Mundial da Saúde), tende a aumentar consideravelmente até 2030, se tornando uma das principais causas de morte (WHO, 2013a). Essa doença se caracteriza por uma disfunção na liberação de insulina ou na ação da mesma causando uma constante hiperglicemia; sua origem se dá por inúmeros fatores (ADA, 2004) e sua classificação é feita em quatro tipos diferentes (NAKATA et al., 2013). A glibenclamida atua no tipo 2 de diabetes mellitus, no qual se encontram os não-insulino-dependentes cujo tratamento se baseia na reeducação alimentar, controle do peso, prática de exercícios físicos e/ou uso de hipoglicemiantes orais (ARAUJO, BRITTO e CRUZ, 2000; RIBEIRO et al., 2006).

Tendo em vista que a absorção de medicamentos administrados por via oral ocorre em duas etapas, sendo estas dissolução e permeabilidade intestinal (SETHIA e SQUILLANTE, 2003; YUKSEL, KANIK e BAYKARA, 2000), tais variáveis influenciam a biodisponibilidade oral (CHILLISTONE e HARDMAN, 2011). Como a GLIB está disposta na classe II do Sistema de Classificação Biofarmacêutico (NETO, 2012; PUIGJANER e PROHENS, 2007; REHDER et al., 2012; SHAH et al., 2013; WEI et al., 2008), apresentando baixa solubilidade e alta permeabilidade, sua absorção se torna limitada pela taxa de dissolução (AMIDON et al., 1995).

A taxa de dissolução pode ser melhorada administrando-se drogas em escalas micro e/ou nanométricas obtendo-se resultados efetivos na melhora de tal taxa e consequentemente na biodisponibilidade do fármaco. (RASENACK, HARTENHAUER e MÜLLER, 2003; VANDANA et al., 2013). 
Assim, foi feita uma avaliação do perfil de dissolução da GLIB cristalizada em diferentes condições experimentais através do processo de precipitação com antissolvente líquido, utilizando etanol como solvente e água como antissolvente, além de TWEEN ${ }^{\circledR} 80$ como aditivo, a fim de verificar a influência dos fatores na taxa de dissolução. As variáveis estudadas e seus respectivos níveis foram: concentração da solução de 0,5 a $2,0 \mathrm{mg}$. $\mathrm{mL}^{-1}$, razão de volume antissolvente/solvente $[\mathrm{A} / \mathrm{S}]$ de 5 a 20, intensidade de agitação de 300 a 1500 rpm e concentração de aditivo de 0,000 a $0,002 \%$ [massa de aditivo/massa de GLIB]. Informações detalhadas sobre o processo de precipitação da GLIB, bem como das condições experimentais utilizadas podem ser encontradas no estudo previamente realizado por Costa (2015).

\section{MATERIAIS E MÉTODOS}

\subsection{Materiais}

Glibenclamida (CAS 10238-21-8) doada pela Fundação para o Remédio Popular (FURP - Guarulhos, SP, Brasil). Nos testes de dissolução foram utilizados tampão fosfato 3,0 e acetonitrila como fase móvel e tampão fosfato pH 7,3 como meio de dissolução.

\subsection{Teste de dissolução}

O teste de dissolução visa identificar o quanto do fármaco se encontra em solução em determinado tempo (ANVISA, 2010a; FILHO, 2011).

Procedimento: Após a desaeração (retirada de gases dissolvidos), verteu-se $900 \mathrm{~mL}$ do meio de dissolução a uma cuba de boro silicato onde a temperatura de $37^{\circ} \mathrm{C} \pm 0,5^{\circ} \mathrm{C}$ foi mantida com o auxílio de um banho termostático (ALPHA RA 8, Lauda). Após a estabilização da temperatura, adicionou-se $5 \mathrm{mg}$ de amostra e iniciou-se a agitação com haste de aço inoxidável (homemade) na forma de pá a $75 \mathrm{rpm}$.

Em seguida, retirou-se amostras de $5 \mathrm{~mL}$ da solução utilizando os intervalos de 10, 20, 30, 40, 50, 60, 90 e 120 minutos, com o intuito de determinar a taxa dissolução das partículas. Posteriormente à retirada de cada volume da solução, repôs-se a mesma quantidade com tampão fosfato 7,3 , sendo cada ensaio feito em duplicata.

As amostras retiradas, foram filtradas com o auxílio de membranas de 0,22 $\mu \mathrm{m}$ (Millipore $^{\circledR}$ ) de porosidade, e analisadas por um cromatógrafo de fase líquida de alta eficiência (Modelo LC-20AT, Shimadzu) provido de detector UV-Visível (Modelo SPDM20A, Shimadzu), coluna C18 de fase reversa Shimadzu (Shim-pack CLC-ODS, de $6 \mathrm{~mm}$ de diâmetro interno e $150 \mathrm{~mm}$ de comprimento) empacotada com sílica ligada ao grupo octadecilsilano, mantida à temperatura ambiente. A fase móvel foi constituída por uma mistura de tampão fosfato $\mathrm{pH} \mathrm{3,0} \mathrm{e} \mathrm{acetonitrila} \mathrm{(30:70)} \mathrm{e} \mathrm{fluxo} \mathrm{de} \mathrm{1,0} \mathrm{mL.min}{ }^{-1}$; foi utilizado o comprimento de onda de $230 \mathrm{~nm}$ e o tempo de retenção da GLIB foi de 5,5 min.

Injetou-se, separadamente, $20 \mu \mathrm{l}$ de cada amostra retirada, registrou-se os cromatogramas e mediu-se as áreas dos picos. Assim, calculou-se a quantidade de GLIB dissolvida por tempo a partir da área do pico de cada amostra comparada com as áreas da curva padrão.

Curva-padrão: Preparou-se a curva padrão a partir de uma solução principal, onde 11 $\mathrm{mg}$ de GLIB foram dissolvidas em $50 \mathrm{~mL}$ de acetonitrila. Dessa solução retirou-se uma 
alíquota de $2,5 \mathrm{~mL}$ que foi então diluída em $50 \mathrm{~mL}$ de tampão fosfato $\mathrm{pH} 7,3$. Com o intuito de obter as concentrações determinadas na curva de calibração, uma segunda diluição foi feita retirando-se alíquotas da solução preparada durante a primeira diluição, colocadas em um balão volumétrico de $10 \mathrm{~mL}$ e diluídas com tampão fosfato $\mathrm{pH} 7,3$, obtendo as concentrações de 0,14 a $8,8 \mu \mathrm{g} \cdot \mathrm{mL}^{-1}\left(\mathrm{R}^{2}=0,9960\right)$.

\section{RESULTADOS E DISCUSSÕES}

Para o processo de precipitação com antissolvente foram realizados nove experimentos gerados pelo "software" Statistica ${ }^{\circledR} 10$, sendo utilizada uma condição experimental diferente em cada experimento. Posteriormente, foi feita a análise de tamanho, bem como a distribuição de tamanho das partículas (DTP) cristalizadas em cada condição experimental.

$\mathrm{Na}$ determinação do perfil de dissolução, foram analisadas as partículas presentes nas condições experimentais 1, 3 e 7, de um planejamento experimental que pode ser visto em Costa (2015) cujos resultados estão dispostos abaixo na Figura 1:

Figura 1: Dissolução de GLIB [\%] versus tempo [min] das condições experimentais 1, 3 e 7.

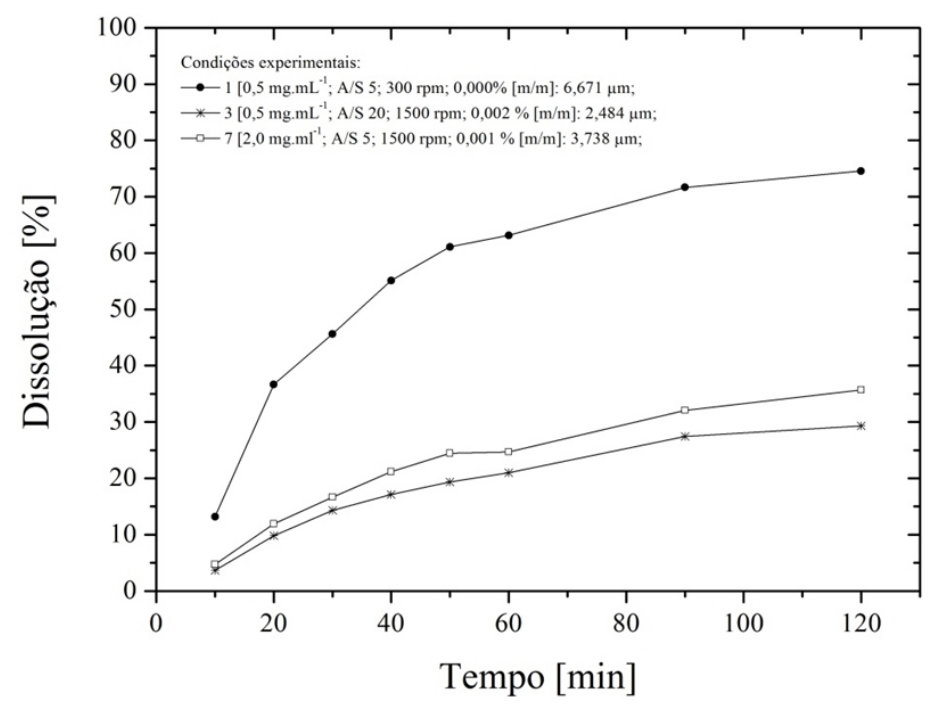

Dentre as condições experimentais utilizadas, a GLIB proveniente da condição 3 exibiu o menor DM $(2,484 \mu \mathrm{m})$ e, devido à isso, previa-se obter maior taxa de dissolução para tais partículas. Entretanto, a maior quantidade de finos, ou seja, partículas menores que $1 \mu \mathrm{m}$, contribuiu para que a GLIB obtida da condição experimental 1, exibisse maior porcentagem de dissolução em menor tempo de análise. As porcentagens em volume das partículas de tamanho entre 0,010 e $0,955 \mu \mathrm{m}$ presentes nas amostras analisadas podem ser observadas na Figura 2, abaixo:

Dentre a faixa granulométrica avaliada, observa-se que a condição 1 exibiu a maior porcentagem de partículas menores que $0,7 \mu \mathrm{m}$, seguida pelas condições experimentais 3 e 7 , respectivamente. Quanto às partículas maiores que $0,7 \mu \mathrm{m}$, a GLIB proveniente da condição experimental 3 exibiu a maior porcentagem de partículas, seguida pelas condições 1 e 7 , respectivamente.

Figura 2: Porcentagem de partículas de acordo com sua granulometria. 

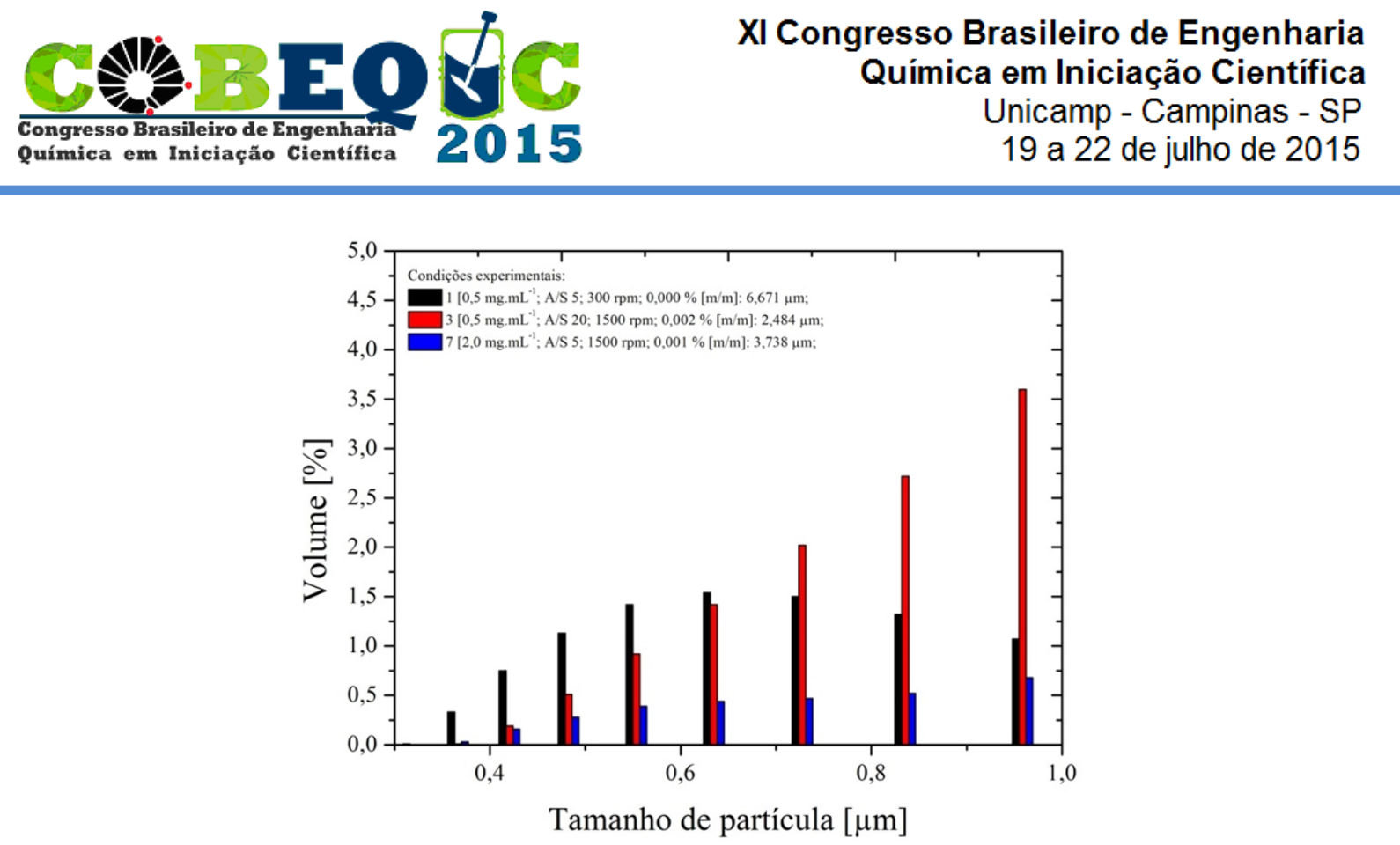

Tendo em vista que partículas de menor tamanho tendem a se dissolver mais rapidamente, a taxa de dissolução na condição experimental 1 é maior quando comparada à condição 3. Vale ressaltar que a GLIB obtida em 7 apresentou uma taxa de dissolução maior que em 3, tendo vista a menor quantidade de partículas de maior granulometria.

Embora não tenham sido encontrados resultados semelhantes na literatura, tal comportamento pode estar relacionado às etapas de nucleação e crescimento dos cristais, as quais devem ocorrer de forma controlada para que seja possível obter partículas de menor diâmetro médio e uma DTP normal.

\section{CONCLUSÃO}

O processo de precipitação com antissolvente líquido é eficiente para produzir partículas em escala micrométrica, tornando a amostra mais homogênea, em relação à distribuição do tamanho das partículas, se comparada à inicial.

Já o teste de dissolução mostra que mesmo que o tamanho médio das partículas seja menor, uma amostra com maior porcentagem de finos terá uma maior taxa de dissolução e conseqüentemente maior biodisponiblidade. Diante disso, pode-se concluir que para se obter maiores porcentagens de dissolução, é preciso produzir partículas que apresentem estreita distribuição granulométrica, porém em escala nanométrica.

\section{REFERÊNCIAS}

ADA (American Diabetes Association). Diagnosis and Classification of Diabetes Mellitus. Diabetes Care, vol. 27, n. 1, p. 5-10, 2004.

AMIDON, G. L.; LENNERNÃS, H.; SHAH, V. P.; CRISON, J. R. A Theoretical Basis for a Biopharmaceutic Drug Classification: The Correlation of in Vitro Drug Product Dissolution and in Vivo Bioavailability. Pharmaceutical Research, v. 12, n. 3, p. 413-420, 1995.

ANVISA (Agência Nacional de Vigilância Sanitária). Farmacopeia Brasileira, 2010a. v. 1. 5 ed. Brasília. Disponível em:< 


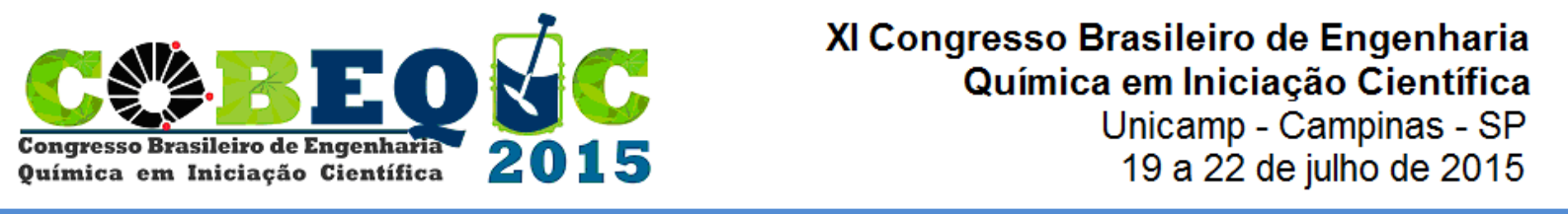

http://www.anvisa.gov.br/hotsite/cd_farmacopeia/pdf/volume1.pdf $>$. Acesso em outubro de 2013.

ARAÚJO, L. M. B.; BRITTO, M. M. dos S.; CRUZ, T. P. da. Tratamento do Diabetes Mellitus do Tipo 2: Novas Opções. Arq. Bras. Endocrinologia \& Metabologia, v. 44, n. 6, p. 509-518, 2000.

CHILLISTONE, S.; HARDMAN, J. Factors affecting drug absorption and distribution. Anaesthesia and Intensive Care Medicine, v. 9, p. 167-171, 2011.

COSTA, CAMILA STEFANNE DIAS. Cristalização do princípio ativo farmacêutico glibenclamida por processo de precipitação com antissolvente líquido. 2015. 100p. Dissertação (Mestrado em Engenharia Química). Departamento de Engenharia Química, Universidade Federal Rural do Rio de Janeiro, Seropédica, 2015.

FILHO, E. Q. da S. Estudo de propriedades físico-químicas da glibenclamida que influem sobre resultados do ensaio de dissolução para medicamento similar e genérico. 2011.91 p. Dissertação (Mestre em Vigilância Sanitária) - Instituto Nacional de Controle de Qualidade em Saúde, Fundação Oswaldo Cruz, Rio de Janeiro, 2011.

NAKATA, B.; ISHIKAWA, T.; AMANO, R.; KIMURA, K.; HIRAKAWA, K. Impact of preoperative diabetes mellitus on clinical outcome after pancreatectomy. International Journal of Surgery, 11, p. 757-761, 2013.

NETO, S. A. de L. Preparação e caracterização analítica de dispersões sólidas cristalinas de glibenclamida, obtidas através de secagem por aspersão. 2012. 102 p. Tese (Doutor em Produtos Naturais e Sintéticos Bioativos) - Centro de Ciências da Saúde, Universidade Federal da Paraíba, João Pessoa, 2012.

PUIGJANER, C.; PROHENS, R. Polimorfismo en la industria farmacêutica. El Farmacéutico, n. 373, p. 58-68, 2007.

RASENACK, N.; HARTENHAUER, H.; MÜLLER, B. W. Microcrystals for dissolution rate enhancement of poorly water-soluble drugs. International Journal of Pharmaceutics, 254, p. 137-145, 2003.

REHDER, S.; SAKMANN, A.; RADES, T.; LEOPOLD, C. S. Thermal degradation of amorphous glibenclamide. European Journal of Pharmaceutics and Biopharmaceutics, 80, p. 203-208, 2012.

RIBEIRO, A. L. P.; POLANCZYK, C. A.; NASCIMENTO, C. A. L. do; NOGUEIRA, J. L. dos S.; CARVALHO, R. S. V. N. de Cadernos de Atenção Básica: Diabetes Mellitus. n. 16, 56 p, 2006. Disponível em:

$<$ http://189.28.128.100/dab/docs/publicacoes/cadernos_ab/abcad16.pdf >. Acesso em novembro de 2013. 


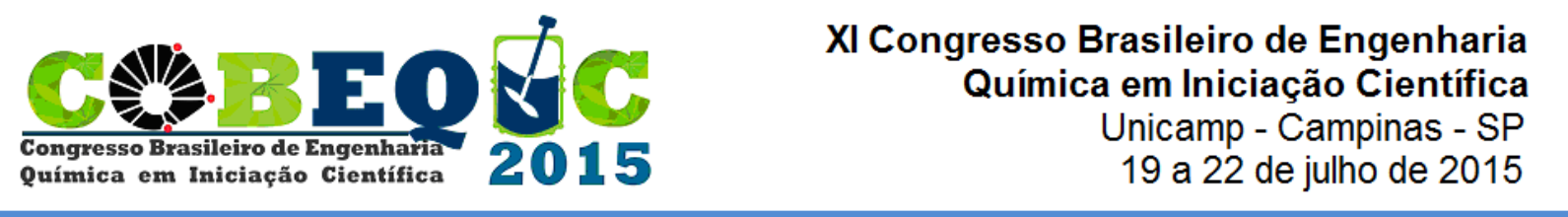

SETHIA, S.; SQUILLANTE, E. Solid Dispersions: Revival with Greater Possibilities and Applications in Oral Drug Delivery. Critical Reviews ${ }^{\text {TM }}$ in Therapeutic Drug Carrier Systems, 20, p. 215-247, 2003.

SHAH, S. R.; PARIKH, R. H.; CHAVDA, J. R.; SHETH, N. R. Application of PlackettBurman screening design for preparing glibenclamide nanoparticles for dissolution enhancement. Powder Technology, 235, p. 405-411, 2013.

VANDANA, K. R.; RAJU, Y. P.; CHOWDARY, V. H.; SUSHMA, M.; KUMAR, N. V. An overview on in situ micronization technique - An emerging novel concept in advanced drug delivery. Saudi Pharmaceutical Journal, 2013.

WEI, H.; DALTON, C.; MASO, M. D.; KANFER, I.; LÖBENBERG, R. Physicochemical characterization of five glyburide powders: A BCS based approach to predict oral absorption. European Journal of Pharmaceutics and Biopharmaceutics, 69, p. 1046-1056, 2008.

World Health Organization (WHO). Diabetes. 2013a. Disponível em:

$<$ http://www.who.int/mediacentre/factsheets/fs312/en/>. Acesso em outubro de 2013.

YUKSEL, N.; KANIK, A. E.; BAYKARA, T. Comparison of in vitro dissolution profiles by ANOVA-based, model-dependent and-independent methods. International Journal of Pharmaceutics, 209, p. 57-67, 2000.

Os autores agradecem à FAPERJ pelo apoio financeiro. 